\title{
Meta-analysis demonstrates safety of pacemaker reutilization
}

Reusing artificial pacemakers carries low risks of infection and device malfunction, according to the first meta-analysis to examine pacemaker recycling on a large scale. Pacemaker reuse might, therefore, be a viable option for overcoming the economic constraints that currently inhibit the implantation of pacemakers in patients with bradycardia or heart block in many low-and-middle-income countries.

This study was conducted by the My Heart-Your Heart group, who hope to collect pacemakers explanted by funeral directors and, after rigorous checking of performance and decontamination, distribute them to low-and-middle-income countries for reimplantation in new recipients. They are currently awaiting FDA approval for their program.

The researchers collated data from 2,270 patients enrolled in 18 trials performed in 13 countries between 1970 and 2010. Average follow-up was $35 \pm 25$ months. The investigators showed a low incidence of adverse effects (infection: $1.97 \%$; malfunction: $0.68 \%$ ) following pacemaker reuse. In the five trials in which control patients received new devices, the risk of infection was not different between the two patient groups but, for the four trials with available data, the risk of pacemaker malfunction was significantly higher in patients with recycled devices (odds ratio 5.80; 95\% CI 1.93-17.47; $P=0.002$ ).

"We believe that post-mortem pacemaker reuse [will] be a safe, feasible, and ethically responsible means of delivering electrophysiological health care to those in great need," comments Dr Timir Baman, lead author of the report.

Gregory B. Lim

Original article Baman, T. S. et al. Safety of Pacemaker Reutilization: A Meta-Analysis with Implications for Underserved Nations. Circ. Arrhythm. Electrophysiol. doi:10.1161/CIRCEP.110.960112 